\title{
A POISSON INTEGRAL FORMULA FOR THE ELLIPSE AND SOME APPLICATIONS
}

\author{
W. C. ROYSTER ${ }^{1}$
}

1. Introduction. Let $E=\left\{(x, y) \mid x^{2} / a^{2}+y^{2} / b^{2}=1\right\}$ be an ellipse with foci at \pm 1 . The ellipse can be represented parametrically by $z=\cos (t-i s)$, where $z=x+i y, s=\tanh ^{-1} b / a, a>b>0$, and $0 \leqq t<2 \pi$. It is known [5] that any function regular inside $E$ can be expanded in a series of Tchebychef polynomials

$$
f(z)=\sum_{n=0}^{\infty} a_{n} T_{n}(z), \quad T_{n}(z)=\cos n(\arccos z),
$$

that converges uniformly interior to $E$.

Consequently, if $U(x, y)$ is any function that is harmonic in $E$, then $U$ can be represented as the real part of some $f$ that is given by (1). We shall use this fact to establish a Poisson integral formula for $U$. We should mention here that the Poisson formula can also be obtained by mapping the ellipse in the $z$-plane onto a rectangle in the $w$-plane thus obtaining $F(w)$ as the transform of $f(z)$ and applying the Cauchy integral formula, using in addition some properties of the Jacobian elliptic function $Z(w)$. We have chosen the method presented in this paper because of its simplicity.

The Poisson formula is readily generalized to the Poisson-Stieltjes formula and to what is commonly called the Herglotz formula for nonnegative harmonic functions. We apply the Poisson-Stieltjes formula to the class $\beta$ of functions with positive real part in $E$ and extend it to find an integral representation for functions regular in $E$ and mapping $E$ onto a domain that is starlike with respect to the origin. Other classes of functions related to $P$ in various ways could also be studied by this method. The coefficient problem for the class of starlike functions as well as other subclasses of functions that are univalent in $E$ and can be related to $\beta$ is studied in [4].

2. Main Theorem. Suppose that $E^{\prime}$ is given by $z=\cos \left(t^{\prime}-i c\right)$, $c<\infty$, and that $s<c$, that is, Int $(E) \subset \operatorname{Int}\left(E^{\prime}\right)$. Also, let $f(z)$ be regular in $E^{\prime}$ and continuous on $E^{\prime}$. If we put $f(z)=U(s, t)+i V(s, t)$ and $a_{n}=\alpha_{n}+i \beta_{n}$, then

Received by the editors May 24, 1962 and, in revised form, March 25, 1963.

1 This research was supported by the National Science Foundation. 


$$
U(s, t)=\alpha_{0}+\sum_{n=1}^{\infty} \alpha_{n} \cosh n s \cos n t-\beta_{n} \sinh n s \sin n t,
$$

which for fixed $s \leqq c$ is the Fourier series expansion for $U(s, t)$. Since we have assumed that $f$ is continuous on $E^{\prime}$ we can replace $s$ by $c$ and get as Fourier coefficients

$$
\alpha_{0}=\frac{1}{2 \pi} \int_{0}^{2 \pi} U\left(c, t^{\prime}\right) d t^{\prime}, \quad \alpha_{n}=\frac{1}{\pi \cosh n c} \int_{0}^{2 \pi} U\left(c, t^{\prime}\right) \cos n t^{\prime} d t^{\prime},
$$

$$
\beta_{n}=-\frac{1}{\pi \sinh n c} \int_{0}^{2 \pi} U\left(c, t^{\prime}\right) \sin n t^{\prime} d t^{\prime}
$$

Substituting formulas (3) into (2) we get

$$
\begin{aligned}
U(s, t)= & \frac{1}{2 \pi} \int_{0}^{2 \pi} U\left(c, t^{\prime}\right) d t^{\prime} \\
+ & \frac{1}{\pi} \int_{0}^{2 \pi} U\left(c, t^{\prime}\right)\left[\sum_{n=1}^{\infty} \frac{\cosh n s}{\cosh n c} \cos n t \cos n t^{\prime}\right. \\
& \left.\quad+\frac{\sinh n s}{\sinh n c} \sin n t \sin n t^{\prime}\right] d t^{\prime},
\end{aligned}
$$

where the summation and integration processes are permitted to be interchanged because of the uniform convergence of the integrand with respect to $t^{\prime}$.

Our task now is to sum the series occurring in (4). Using the identities for $\cos n t \cos n t^{\prime}$ and $\sin n t \sin n t^{\prime}$ we get

$$
\sum_{n=1}^{\infty} k_{n}=\sum_{n=1}^{\infty} \frac{1}{\sinh 2 n c}\left[\sinh n(c-s) \cos n\left(t+t^{\prime}\right)\right.
$$

$$
\left.+\sinh n(c+s) \cos n\left(t-t^{\prime}\right)\right\rfloor
$$

$$
\begin{aligned}
=\sum_{n=1}^{\infty} \frac{1}{\sinh 2 n c} \operatorname{Im}\left\{\sin n\left[\left(t+t^{\prime}\right)+i(c-s)\right]\right. \\
\left.\quad-\sin n\left[\left(t-t^{\prime}\right)-i(c+s)\right]\right\},
\end{aligned}
$$

where $k_{n}$ stands for the summand in (4). Let $c=\frac{1}{2} \log 1 / q$, then $\sinh 2 n c$ $=\frac{1}{2}\left(q^{-n}-q^{n}\right)$ and (5) becomes

(6) $\sum_{n=1}^{\infty} k_{n}=2 \operatorname{Im}\left\{\sum_{n=1}^{\infty} \frac{q^{n}}{1-q^{2 n}}\left(\sin n\left[\left(t+t^{\prime}\right)+i(c-s)\right]\right.\right.$

$$
\left.\left.-\sin n\left[\left(t-t^{\prime}\right)-i(c+s)\right]\right)\right\} \text {. }
$$


This function is easily given in terms of the Jacobi zeta function [ 1 , pp. 405, 419], given by the series

$$
Z(u)=\frac{2 \pi}{K} \sum_{n=1}^{\infty} \frac{q^{n}}{1-q^{2 n}} \sin \frac{n \pi u}{K}, \quad q=e^{-K^{\prime} / K},
$$

valid for $|\operatorname{Im}(u / K)|<2 \operatorname{Re}\left(K^{\prime} / K\right)$, which is periodic and satisfies the relation $Z(u+2 K)=Z(u)$ and where $K$ and $K^{\prime}$ are the customary periods associated with the Jacobi $s n$ function. Since $f(z)$ is to be single-valued we set $K=\pi$. Hence (6) can be written as

$$
\begin{aligned}
\sum_{n=1}^{\infty} k_{n} & =\operatorname{Im}\left\{Z\left[\left(t+t^{\prime}\right)+i(c-s)\right]-Z\left[\left(t-t^{\prime}\right)-i(c+s)\right]\right\} \\
& =\operatorname{Im}\left\{Z\left[(t-i s)+\left(t^{\prime}+i c\right)\right]-Z\left[(t-i s)-\left(t^{\prime}+i c\right)\right]\right\} .
\end{aligned}
$$

Substituting (7) into (4) we finally obtain

$$
\begin{gathered}
U(s, t)=\frac{1}{2 \pi} \int_{0}^{2 \pi} U\left(c, t^{\prime}\right) d t^{\prime} \\
(8)+\int_{0}^{2 \pi} \operatorname{Re}\left\{\frac{1}{i \pi}\left[Z\left(\cos ^{-1} z+t^{\prime}+i c\right)-Z\left(\cos ^{-1} z-t^{\prime}-i c\right)\right]\right\} \cdot U\left(c, t^{\prime}\right) d t^{\prime},
\end{gathered}
$$

where $z=\cos (t-i s)$.

Let

$$
\text { (9) } K(z, \bar{\zeta})=1+\frac{2}{i}\left[Z\left(\cos ^{-1} z+t^{\prime}+i c\right)-Z\left(\cos ^{-1} z-t^{\prime}-i c\right)\right] \text {, }
$$

where $\zeta=\cos \left(t^{\prime}-i c\right)$. By direct computation we can show that $\operatorname{Re}\{K(z, \bar{\zeta})\}$ is harmonic in $E^{\prime}$. Hence its analytic completion is regular in $E^{\prime}$. The function $K(z, \bar{\zeta})$ could equally well be given in terms of the Weierstrass zeta function since the two zeta functions are linearly related after a transformation of variables [1].

We are now in a position to state our theorem.

TheOREM 1. Let $U$ be a function harmonic in the ellipse $E^{\prime}$ and continuous on $E^{\prime}$. Then at each point $z$ interior to $E^{\prime}, U$ is represented by

$$
U(z)=\frac{1}{2 \pi} \int_{0}^{2 \pi} U\left(c, t^{\prime}\right) \operatorname{Re}\{K(z, \bar{\zeta})\} d t^{\prime},
$$

where $K(z, \bar{\zeta})$ is given by (9) and $\zeta=\cos \left(t^{\prime}-i c\right)$.

Formula (10) is the Poisson integral formula for the ellipse.

It should be remarked that continuity on $E^{\prime}$ is not necessary. This 
condition can be weakened to piecewise continuity leaving the integral in its present form.

The Poisson integral representation (10) can be generalized further to a Stieltjes integral representation in much the same manner as in the case of the unit circle. We shall not go through the details but refer the reader to the two books: M. Heins, Selected topics in the classical theory of functions of a complex variable, pp. 66-69, Holt, New York, 1962, and G. Evans, The logarithmic potential, Amer. Math. Soc. Colloq. Publ. Vol. 6, Chapter 2, Amer. Math. Soc., Providence, R. I., 1927.

Let us now study some properties of $K(z, \bar{\zeta})$. We have already stated that $K(z, \bar{\zeta})$ is regular in $E^{\prime}$. However as $z \rightarrow \zeta, s<c, K(z, \bar{\zeta}) \rightarrow \infty$, hence $K(z, \bar{\zeta})$ has a pole at $z=\zeta, \zeta$ a fixed point on $E^{\prime}$. Furthermore $K(z, \bar{\zeta})$ has positive real part in $E^{\prime}$. Indeed, writing

$$
\operatorname{Re}\{K(z, \bar{\zeta})\}=1+2 \sum_{n=1}^{\infty} k_{n}=1+2 \sum_{n=1}^{\infty} P_{n}+Q_{n}
$$

where $P_{n}$ and $Q_{n}$ represent the first and second terms of the summand in (5), respectively, we have for $s=c$ that $\sum_{n=1}^{\infty} P_{n}=0$. However, we need to take a closer look at the rest of the expression in (5), that is, we write

$$
\begin{aligned}
1+2 \sum_{n=1}^{\infty} Q_{n}= & 1+2 \sum_{n=1}^{\infty}\left(\frac{e^{s}}{e^{c}}\right)^{n} \frac{1-\left(e^{-c-s}\right)^{2 n}}{1-e^{-4 n c}} \cos n\left(t-t^{\prime}\right) \\
= & 1+2 \sum_{n=1}^{\infty}\left(\frac{e^{s}}{e^{c}}\right)^{n}\left[1+\frac{e^{-4 n c}-\left(e^{-c-s}\right)^{2 n}}{1-e^{-4 n c}}\right] \cos n\left(t-t^{\prime}\right) \\
= & \frac{1-\left(\frac{e^{s}}{e^{c}}\right)^{2}}{1-2\left(\frac{e^{s}}{e^{c}}\right) \cos \left(t-t^{\prime}\right)+\left(\frac{e^{s}}{e^{c}}\right)^{2}} \\
& +2 \sum_{n=1}^{\infty}\left(\frac{e^{s}}{e^{c}}\right)^{n} \frac{e^{-4 n c}-\left(e^{-c-s}\right)^{2 n}}{1-e^{-4 n c}} \cos n\left(t-t^{\prime}\right)
\end{aligned}
$$

which gives for $s=c, t \neq t^{\prime}$, zero boundary values for $1+2 \sum Q_{n}$. Hence $\operatorname{Re}\{K(z, \bar{\zeta})\} \geqq 0$ for $z$ in $E^{\prime}$. The function $K(z, \bar{\zeta})$ has another interesting property that is easily noted if we write it in terms of Tchebychef polynomials. Written in terms of these polynomials $K(z, \bar{\zeta})$ becomes 


$$
\begin{aligned}
K(z, \bar{\zeta}) & =1+\frac{2}{i} \sum_{n=1}^{\infty} \frac{1}{\sinh 2 n c}\left(\sin n\left[(t-i s)+\left(t^{\prime}+i c\right)\right]\right. \\
& =1+\frac{4}{i} \sum_{n=1}^{\infty} \frac{\sin n\left(t^{\prime}+i c\right)}{\sinh 2 n c} \cos n(t-i s) \\
& =1+\sum_{n=1}^{\infty}\left(\alpha_{n}+i \beta_{n}\right) T_{n}(z),
\end{aligned}
$$

where $\alpha_{n}=2 \cos n t^{\prime} \cosh n c$ and $\beta_{n}=-2 \sin n t^{\prime} \sinh n c$. We see immediately that $\alpha_{n}$ and $\beta_{n}$ satisfy the equation

$$
\left|\alpha_{n} \cosh n c+i \beta_{n} \sinh n c\right|=2 \text {. }
$$

It has been shown in an earlier paper [3] that if $\operatorname{Re}\{f(z)\} \geqq 0, f$ given by (1), $a_{0}=1$, in an ellipse with foci at \pm 1 and semi axes $a$ and $b$, $a+b=R$, then $\left(^{*}\right) \alpha_{n}^{2}\left(R^{n}+R^{-n}\right)^{2}+\beta_{n}^{2}\left(R^{n}-R^{-n}\right)^{2} \leqq 16$. It was shown that $\left({ }^{*}\right)$ was best possible by constructing for each $n$ a function satisfying equality in $\left(^{*}\right)$. If in (13) we set $e^{c}=R$ we get equality in $\left(^{*}\right)$. Hence $K(z, \bar{\zeta})$ is the extremal function for $\left(^{*}\right)$.

3. Applications. Let $\beta$ be the class of functions regular in the ellipse $E^{\prime}$ that map $E^{\prime}$ onto a domain in the right half plane, i.e., $\operatorname{Re}\{f(z)\}$ $\geqq 0$ for $z \in \operatorname{Int}\left(E^{\prime}\right)$, and that are normalized by the condition $(1 / 2 \pi) \int_{0}^{2 \pi} f(z) d t=1, z=\cos (t-i s)$. We have the following representation for members of $P$.

Theorem 2. Any $f \in P$ can be represented by a Poisson-Stieltjes integral of the form

$$
f(z)=\int_{0}^{2 \pi} K(z, \bar{\zeta}) d \psi\left(t^{\prime}\right)
$$

where $z=\cos (t-i s)$ belongs to the interior of $E^{\prime}, \zeta=\cos \left(t^{\prime}-i c\right)$, and $\psi\left(t^{\prime}\right)$ is a real-valued nondecreasing function defined in $0 \leqq t^{\prime} \leqq 2 \pi$ and having total variation one.

Proof. For fixed $s, 0<s<c$, set

$$
\psi\left(t^{\prime}, s\right)=\frac{1}{2 \pi} \int_{0}^{t^{\prime}} \operatorname{Re}\{f(z)\} d t, \quad z=\cos \left(t^{\prime}-i s\right) .
$$

The function $\psi\left(t^{\prime}, s\right)$ is a nondecreasing function of $t^{\prime}$, since $\operatorname{Re}\{f(z)\}$ $\geqq 0$ for $z \in \operatorname{Int}\left(E^{\prime}\right)$. The total variation is given by

$$
\psi(2 \pi, s)=\frac{1}{2 \pi} \int_{0}^{2 \pi} \operatorname{Re}\{f(z)\} d t=1 .
$$


Consider the family $F$ of functions $\left\{\psi\left(t^{\prime}, s\right)\right\}$. According to the Helly selection principle [Natanson, Theory of functions of a real variable, Ungar, New York, 1955] we can find a sequence of functions $\left\{\psi\left(t^{\prime}, s_{k}\right)\right\}, s_{k} \rightarrow c$ as $k \rightarrow \infty$, that converges pointwise to a function $\psi\left(t^{\prime}, c\right)=\psi\left(t^{\prime}\right)$ which is nondecreasing and of total variation one. Now consider the integral

$$
\begin{aligned}
\int_{0}^{2 \pi} & \sin n\left(t^{\prime}+i c\right) d \psi\left(t^{\prime}, s_{k}\right) \\
= & \frac{1}{4 \pi}\left\{\int_{0}^{2 \pi} \sin n\left(t^{\prime}+i c\right) f\left(\cos \left(t^{\prime}-i s_{k}\right)\right) d t^{\prime}\right. \\
& \left.\quad+\int_{0}^{2 \pi}\left[\sin n\left(t^{\prime}-i c\right) f\left(\cos \left(t^{\prime}-i s_{k}\right)\right)\right]-d t^{\prime}\right\} \\
= & \frac{i}{4}\left[a_{n} \sinh n\left(c+s_{k}\right)+\bar{a}_{n} \sinh n\left(c-s_{k}\right)\right],
\end{aligned}
$$

$n=1,2,3, \cdots$, where we have used the fact that $f\left(\cos \left(t^{\prime}-i s_{k}\right)\right)$ $=\sum_{n=0}^{\infty} a_{n} \cos n\left(t^{\prime}-i s_{k}\right)$ is uniformly convergent with respect to $t^{\prime}$. Hence

$$
\begin{aligned}
& \lim _{s_{k} \rightarrow c} \int_{0}^{2 \pi} \sin n\left(t^{\prime}+i c\right) d \psi\left(t^{\prime}, s_{k}\right) \\
& \quad=\int_{0}^{2 \pi} \sin n\left(t^{\prime}+i c\right) d \psi\left(t^{\prime}\right)=\frac{i}{4} a_{n} \sinh 2 n c,
\end{aligned}
$$

so that $a_{n}=(4 / i \sinh 2 n c) \int_{0}^{2 \pi} \sin n\left(t^{\prime}+i c\right) d \psi\left(t^{\prime}\right), n=1,2, \cdots$, $a_{0}=1+i k_{1}, k_{1}$ real. Substituting these values of $a_{n}$ into (1) and employing (12) we get

$$
f(z)=\int_{0}^{2 \pi} K(z, \bar{\zeta}) d \psi\left(t^{\prime}\right)+i k_{1},
$$

where $k_{1}$ is a real constant. The proof of the theorem will be complete upon the determination of the constant $k_{1}$. From the normalization conditions we have, for $z=\cos (t-i s)$,

$$
\begin{aligned}
1 & =\frac{1}{2 \pi} \int_{0}^{2 \pi} f(z) d t=\frac{1}{2 \pi} \int_{0}^{2 \pi}\left[\int_{0}^{2 \pi} K(z, \bar{\zeta}) d \psi\left(t^{\prime}\right)+i k_{1}\right] d t \\
& =\frac{1}{2 \pi} \int_{0}^{2 \pi}\left[\int_{0}^{2 \pi} K(z, \bar{\zeta}) d t\right] d \psi\left(t^{\prime}\right)+i k_{1} .
\end{aligned}
$$

The function $Z(u)$ is also defined as $\Theta^{\prime}(u) / \Theta(u)$, where $\Theta(u)$ is the 
Jacobi theta function, periodic of period $2 K=2 \pi$ and also even [1, p. 405]. Substituting (9) into (18) and integrating yields

$$
1=\frac{1}{2 \pi} \int_{0}^{2 \pi}\left[t+\frac{2}{i} \log \frac{\Theta\left(t-i s+t^{\prime}-i c\right)}{\Theta\left(t-i s-t^{\prime}-i c\right)}\right]_{0}^{2 \pi} d \psi\left(t^{\prime}\right)+i k_{1}=1+i k_{1} .
$$

Hence $k_{1}=0$.

We can use Theorem 2 to obtain some extremal properties for functions belonging to $P$. However, before we look at these extremal problems let us first consider any function $f(z)$ that is regular in an ellipse $E$ with foci at \pm 1 and semi-axes satisfying $a+b=R=e^{s}$. Such a function is given by (1). Let $A(E)$ be the area of the map of $E$ under the transformation $w=f(z)$. We have the following result.

TheOREM 3. Let $f(z)$ be given by (1) and $A(E)$ be the area of $D_{E}$, the map of $E$ by $w=f(z)$. Then

$$
A(E)=\frac{\pi}{2} \sum_{n=1}^{\infty} n\left|a_{n}\right|^{2} \sinh 2 n s .
$$

If $D_{E}$ is not schlicht then $A(E)$ represents the area of the multiply covered domain.

Proof. The area of $D_{E}$ is given by the formula $A(E)$ $=\iint_{E}\left|f^{\prime}(z)\right|^{2} d x d y$. By an application of Green's theorem we get $A(E)$ $=(1 / 2 i) \int_{\Gamma} f^{\prime}(z)[f(z)]-d z$, where $\Gamma$ is the boundary of $E$. Hence

$$
A(E)=\frac{1}{2 i} \int_{\Gamma}\left(\sum_{n=0}^{\infty} n a_{n} U_{n-1}(z) \cdot \sum_{m=0}^{\infty}\left[a_{m} T_{m}(z)\right]^{-}\right) d z,
$$

where $U_{n-1}(z)$ is the Tchebychef polynomial of the second kind, $U_{n-1}(z)=\sin n(t-i s)\left(1-z^{2}\right)^{1 / 2}$. It is easy to verify that $(1 / 2 i) \int_{\Gamma} U_{n-1}(z)\left[T_{m}(z)\right]-d z=0,(\pi / 2) \sinh 2 n s$, for $m \neq n, m=n$, respectively. This orthogonality property immediately yields (19).

If $f \in P$ and $E$ is any ellipse interior to $E^{\prime}$ and confocal with $E^{\prime}$ the following theorem gives an upper and lower bound for $A(E)$, the area of the map of $E$ under $w=f(z)$, in terms of $s$ and $c$, or in terms of $R$ and $R^{\prime}$.

TheOREM 4. Let $f \in P$ then

$$
2 \pi \sum_{n=1}^{\infty} n \frac{\sinh 2 n s}{\cosh ^{2} n c} \leqq A(E) \leqq 2 \pi \sum_{n=1}^{\infty} n \frac{\sinh 2 n s}{\sinh ^{2} n c} .
$$

The left hand inequality is sharp, as is shown by $K(z, \bar{\zeta})$, with $t^{\prime}=0$.

Proof. Since $f \in \odot$ we have 


$$
\begin{aligned}
A(E) & =\frac{1}{2 i} \int_{\Gamma}\left(\int_{0}^{2 \pi} \frac{\partial}{\partial z} K(z, \bar{\zeta}) d \psi\left(t^{\prime}\right)\right)\left(\int_{0}^{2 \pi}[K(z, \bar{\zeta})]-d \psi\left(t^{\prime}\right)\right) d z \\
& =\int_{0}^{2 \pi} \int_{0}^{2 \pi}\left(\frac{1}{2 i} \int_{\Gamma} K^{\prime}(z, \bar{\zeta})[K(z, \bar{\zeta})]-d z\right) d \psi\left(t^{\prime}\right) d \psi\left(t^{\prime}\right) .
\end{aligned}
$$

Substituting for $K(z, \bar{\zeta})$ from (12), we have

(23) $A(E)=\int_{0}^{2 \pi} \int_{0}^{2 \pi} 8 \pi \sum_{n=1}^{\infty} \frac{n\left|\sin n\left(t^{\prime}+i c\right)\right|^{2}}{\sinh ^{2} 2 n c} \sinh 2 n s d \psi\left(t^{\prime}\right) d \psi\left(t^{\prime}\right)$.

Applying the inequality $\sinh ^{2} n c \leqq\left|\sin n\left(t^{\prime}+i c\right)\right|^{2} \leqq \cosh ^{2} n c$ and using the fact that the total variation is equal to one yields the inequalities (21).

Continuing in the same trend let $L(\Gamma)$ be the length of the map of $\Gamma$ effected by $w=f(z), f \in P$.

\section{THEOREM 5.}

$$
L(\Gamma) \leqq 8 \sum_{n=1}^{\infty} n \frac{\cosh n s}{\sinh n c} E\left(\frac{1}{\cosh n s}\right)<4 \pi \sum_{n=1}^{\infty} \frac{\cosh n s}{\sinh n c},
$$

where $E(\gamma)=\int_{0}^{1}\left(1-\gamma^{2} x^{2}\right)^{-1 / 2}\left(1-x^{2}\right)^{1 / 2} d x$ is the Jacobian complete elliptic integral of the second kind.

Proof. The value of $L(\Gamma)$ is given by the formula

$$
\begin{aligned}
L(\Gamma) & =\int_{\Gamma}\left|f^{\prime}(z)\right||d z|=\int_{\Gamma}\left|\int_{0}^{2 \pi} K^{\prime}(z, \bar{\zeta}) d \psi\left(t^{\prime}\right)\right||d z| \\
& \leqq \int_{0}^{2 \pi}\left(\int_{\Gamma}\left|K^{\prime}(z, \bar{\zeta})\right||d z|\right) d \psi\left(t^{\prime}\right) .
\end{aligned}
$$

Setting $z=\cos (t-i s), \zeta=\cos \left(t^{\prime}-i c\right)$ and substituting for $K(z, \bar{\zeta})$ from (12) gives

$$
\begin{aligned}
& \int_{\Gamma}\left|K^{\prime}(z, \xi)\right||d z| \\
& \quad \leqq \int_{0}^{2 \pi} 4 \sum_{n=1}^{\infty} n \frac{\left|\sin n\left(t^{\prime}+i c\right)\right|}{\sinh 2 n c}|\sin n(t-i s)| d t .
\end{aligned}
$$

To evaluate $\int_{0}^{2 \pi}|\sin n(t-i s)| d t$, set $v=\cos n t, \gamma=\cosh n s$, then

$$
\begin{aligned}
& \int_{0}^{2 \pi}\left[\sin ^{2} n t \cosh ^{2} n s+\cos ^{2} n t \sinh ^{2} n s\right]^{1 / 2} d t \\
& \quad=4 \int_{0}^{1}\left(\gamma^{2}-v^{2}\right)^{1 / 2}\left(1-v^{2}\right)^{-1 / 2} d v=4 \gamma E\left(\frac{1}{\gamma}\right) .
\end{aligned}
$$


The inequality in the theorem is obtained upon substituting this value into (25) and noting that $\left|\sin n\left(t^{\prime}+i c\right)\right| \leqq \cosh n c$.

The final application we shall make of Theorem 2 is to obtain an integral representation for the family of functions that are starlike in $E^{\prime}$. A necessary and sufficient condition that a function $f(z)$ regular in $E^{\prime}$ map $E^{\prime}$ onto a domain starlike with respect to the origin is

$$
\operatorname{Re}\left\{-i\left(1-z^{2}\right)^{1 / 2} \frac{f^{\prime}(z)}{f(z)}\right\} \geqq 0,
$$

where the determination of $\left(1-z^{2}\right)^{1 / 2}$ is chosen so that at $z=0$, $\left(1-z^{2}\right)^{1 / 2}=1$. The starlikeness condition (26), as well as the corresponding convexity condition, is given in [2].

THEOREM 6. Let $f(z)$ be regular in $E^{\prime}$ and map $E^{\prime}$ onto a domain that is starlike with respect to the origin, then there exists a nondecreasing function $\psi\left(t^{\prime}\right), 0 \leqq t^{\prime}<2 \pi$ of total variation one such that

$$
\left(z^{2}-1\right)^{1 / 2} \frac{f^{\prime}(z)}{f(z)}=-\int_{0}^{2 \pi} K(z, \bar{\zeta}) d \psi\left(t^{\prime}\right)+i k_{2},
$$

where $k_{2}$ is a real constant determined by the normalization conditions.

The proof follows immediately from (26) and Theorem 2.

Suppose $f(z)$ is starlike with respect to the origin and $f(1)=1$, then

$$
\int_{1}^{z} \frac{f^{\prime}(z)}{f(z)} d z=\int_{0}^{2 \pi}\left(\int_{1}^{z} K(z, \bar{\zeta}) \frac{-d z}{\left(1-z^{2}\right)^{1 / 2}}\right) d \psi\left(t^{\prime}\right)+i k_{2} \cos ^{-1} z,
$$

$z \in E^{\prime}$, where the path of integration does not pass through the origin. Integrating (28), after substituting for $K(z, \bar{\zeta})$, we get

$$
\begin{aligned}
\log f(z)= & \int_{0}^{2 \pi}\left[\cos ^{-1} z+\frac{2}{i} \log \frac{\Theta\left(\cos ^{-1} z+t^{\prime}+i c\right)}{\Theta\left(\cos ^{-1} z-t^{\prime}-i c\right)}\right] d \psi\left(t^{\prime}\right) \\
& +i k_{2} \cos ^{-1} z .
\end{aligned}
$$

Consider next a complete circuit about the origin starting at $z=1$, such a circuit exists since $c>0$. The $\Theta$ function is periodic of period $2 \pi$ and $f(z)$ is single-valued, hence the change in the argument is given by

$$
2 \pi i=\int_{0}^{2 \pi} 2 \pi d \psi\left(t^{\prime}\right)+2 \pi i k_{2}
$$

which implies that $k_{2}=1$. Rewriting (28) gives a final representation 


$$
\begin{aligned}
f(z)= & \left(z+i \sqrt{ }\left(1-z^{2}\right)\right) \\
& \cdot \exp \int_{0}^{2 \pi}\left(\cos ^{-1} z+\frac{2}{i} \log \frac{\Theta\left(\cos ^{-1} z+t^{\prime}+i c\right)}{\Theta\left(\cos ^{-1} z-t^{\prime}-i c\right)}\right) d \psi\left(t^{\prime}\right) .
\end{aligned}
$$

As has been previously remarked, this same procedure can be applied to other classes of functions that are related to $P$.

\section{REFERENCES}

1. E. T. Copson, An introduction to the theory of functions of a complex vairable, Clarendon Press, Oxford, 1935.

1. E. T. Copson, An introduction to the theory of functions in a complex variable, Oxford, 1935.

2. W. C. Royster, Convexity and starlikeness of analytic functions, Duke Math. J. 19 (1952), 447-457.

3. - Functions having positive real part in an ellipse, Proc. Amer. Math. Soc. 10 (1959), 266-269.

4. - Coefficient problems for functions regular in an ellipse, Duke Math. J. 26 (1959), 361-372.

5. J. L. Walsh, Note on the orthogonality of Tchebycheff polynomials on confocal ellipses, Bull. Amer. Math. Soc. 40 (1934), 84-88.

The Institute for Advanced Study and

THE UNIVERSITY OF KENTUCKY 